\title{
Nationwide Multicenter Study for Overlaps of Common Functional Gastrointestinal Disorders in Korean Patients With Constipation
}

\begin{abstract}
Kyung Sik Park, ${ }^{1}$ Sam Ryong Jee, ${ }^{2}$ Bong Eun Lee, ${ }^{3}$ Kyoung Sup Hong, ${ }^{4}$ Jeong Eun Shin, ${ }^{5}$ Soo-Young Na, ${ }^{6}$ Joong Goo Kwon, ${ }^{7}$ Suck Chei Choi ${ }^{8 *}$ Yong Sung Kim, ${ }^{9}$ Hyun Seok Lee, ${ }^{10}$ Tae Hee Lee, ${ }_{11}$ Kyeong Ok Kim, $^{12}$ Jongkyoung Choi, ${ }^{13}$ Hee Seok Moon, ${ }^{14}$ Yeon Soo Kim, ${ }_{15}^{15}$ Moo In Park, ${ }^{16}$ Soo Jung Park, ${ }^{17}$ Seon-Young Park, ${ }^{18}$ Sung Noh Hong, ${ }^{19}$ and Constipation Research Group of Korean Society of Neurogastroenterology and Motility

${ }^{1}$ Department of Internal Medicine, Keimyung University School of Medicine, Daegu, Korea; ${ }^{2}$ Inje University College of Medicine, Busan, Korea; ${ }^{3}$ Pusan National University School of Medicine, Busan, Korea; ${ }^{4}$ Seoul National University College of Medicine, Seoul, Korea; ${ }^{5}$ Dankook University College of Medicine, Cheonan, Chungnam, Korea; ${ }^{6} J e j u$ National University School of Medicine, Jeju, Korea; ${ }^{7}$ Catholic University of Daegu School of Medicine, Daegu, Korea; ${ }^{8}$ Wonkwang University School of Medicine, Iksan, Jeonbuk, Korea; ${ }^{9}$ Department of Internal Medicine and Wonkwang Digestive Disease Research Institute, Wonkwang University Sanbon Hospital, Gunpo, Gyeonggi-do, Korea; ${ }^{10}$ Kyungpook National University School of Medicine, Daegu, Korea; ${ }^{11}$ Soonchunhyang University College of Medicine, Seoul, Korea; ${ }^{12}$ Yeungnam University College of Medicine, Daegu, Korea; ${ }^{13}$ National Medical Center, Seoul, Korea; ${ }^{14}$ Chungnam National University School of Medicine, Daejeon, Korea; ${ }^{15} \mathrm{Hallym}$ University College of Medicine, Chuncheon, Gangwon-do, Korea; ${ }^{16}$ Kosin University College of Medicine, Busan, Korea; ${ }^{17}$ Yonsei University College of Medicine, Seoul, Korea; ${ }^{18}$ Chonnam National University Medical School, Gwangju, Korea; and ${ }^{19}$ Sungkyunkwan University School of Medicine, Seoul, Korea
\end{abstract}

\section{Background/Aims}

In spite of increased concerns about the overlaps among the various functional gastrointestinal disorders (FGIDs), studies for the overlap between constipation and other common FGIDs are rare. Therefore, we investigated the patterns of overlaps between constipation and other common FGIDs.

Methods

This study was designed as a prospective nationwide multi-center questionnaire study using Rome III questionnaires for functional dyspepsia (FD), irritable bowel syndrome (IBS), and functional constipation (FC), as well as various questionnaires about patients' information, degree of symptoms, and quality of life. For the evaluation of gastroesophageal reflux disease (GERD), GERD-Q was used.

\section{Results}

From 19 centers, 759 patients with constipation were enrolled. The proportions of FC and IBS subtypes of constipation (IBS-C) were $59.4 \%$ and $40.6 \%$, respectively. Among them, $492(64.8 \%)$ showed no overlap. One hundred and thirty-six patients (17.9\%) presented overlapping GERD, and 80 patients (10.5\%) presented overlapping FD. Fifty one (6.7\%) of patients were overlapped by both GERD and FD. Coincidental herniated nucleus pulposus (HNP) $(P=0.026)$ or pulmonary diseases $(P=0.034)$, reduced fiber intake $(P=$ $0.013)$, and laxative use $(P<0.001)$ independently affected the rate of overlaps. These overlapping conditions negatively affected the constipation-associated quality of life, general quality of life, and degree of constipation.

\section{Conclusions}

The overlap of GERD or FD was common in patients with constipation. Coincidental HNP or pulmonary diseases, reduced fiber intake, and laxatives use were found to be independent associated factors for overlapping common FGIDs in Korean patients with constipation. (J Neurogastroenterol Motil 2017;23:569-577)

Key Words

Constipation; Dyspepsia; Gastroesophageal reflux; Overlapping

Received: March 1, 2017 Revised: May 5, 2017 Accepted: July 12, 2017

(.) This is an Open Access article distributed under the terms of the Creative Commons Attribution Non-Commercial License (http://creativecommons. org/licenses/by-nc/4.0) which permits unrestricted non-commercial use, distribution, and reproduction in any medium, provided the original work is properly cited.

*Correspondence: Suck Chei Choi, MD

Department of Gastroenterology, Wonkwang University Hospital, 895 Muwang-ro, Iksan, Jeollabuk-do 54538, Korea Tel: +82-63-859-2563, Fax: +82-63-855-2025, E-mail: medcsc@wku.ac.kr 


\section{Introduction}

Functional gastrointestinal disorders (FGIDs) are variable combinations of chronic and recurrent gastrointestinal (GI) symptoms in which the identification of underlying mechanisms is not easy. Because there is no objective marker for the diagnosis of FGIDs, the diagnosis is usually based on the patients' symptoms. In adults, FGIDs have been categorized into six groups on the assumption that specific symptoms are related to specific anatomic regions. ${ }^{1,2}$ In Rome III and Rome IV classifications, each disorder is defined exclusively by specific criteria, and this clear definition of each disorder helps maintain homogeneity in research fields. However, in clinical situations, many patients complain of an overlap of 2 or more disorders. Considering common pathophysiologic factors such as dysmotility, visceral hypersensitivity, and gut-brain axis, these overlaps are not strange. ${ }^{3,4}$ In fact, recently, some reports about overlap among functional dyspepsia (FD), gastroesophageal reflux disease (GERD), and irritable bowel syndrome (IBS) have been published. ${ }^{5-11}$ Although there have been increased concerns about the overlap among the various FGIDs, studies of overlap between constipation and other common FGIDs are rare. Therefore, in this study, we investigated the overlap of constipation and other common FGIDs, as well as their associated risk factors. We then investigated how overlap of these conditions affects the quality of life in patients with constipation.

\section{Materials and Methods}

\section{Design}

This study was designed as a non-interventional, prospective, cross-sectional, nationwide multi-center questionnaire study. The protocol of this study was reviewed and approved by the institutional review boards of each institution, including the Institutional Review Board of the Keimyung University Dongsan Medical Center (IRB No. 2013-10-017-002).

\section{Subjects}

Consecutive adult patients (age of 20 or older) who visited outpatient clinics in 19 hospitals complaining of constipation from January 2014 to February 2015 were considered as subjects. The list of 19 participated institutions is presented in the Supplementary Table.

\section{Questionnaires}

If patients agreed to participate in this study, they were requested to sign the patient authorization form and to complete the Korean version of the preliminary evaluation questionnaire, which consisted of 13 items. This questionnaire was conducted by the Korean Society of Neurogastroenterology and Motility (KSNM) based on Rome III criteria for IBS and functional constipation (FC). ${ }^{12,13}$ The patients who fulfilled the Rome III criteria for IBSconstipation (IBS-C) or FC were enrolled in this study after the exclusion of organic disorders such as acute abdomen, intestinal obstruction, intestinal pseudo-obstruction, and malignancy.

Enrolled patients were requested to complete the case report form, which included several demographic data, residence, smoking history, drinking history, history of present illness, history of medication, and preference of specific foods. The presence of diabetes mellitus (DM) or hypertension was assessed with closed questions about each disease. However, the presence of other diseases, such as pulmonary disease, cerebrovascular accident (CVA), or herniated nucleus pulposus (HNP), were investigated with open questions. The presence of GERD was assessed with diagnostic questionnaire for gastro-esophageal reflux disease (GERD-Q) which consists of 6 items. ${ }^{14}$ Furthermore, the presence of FD was evaluated with the Korean version of questionnaire, which consists of 11 items concerning upper GI symptoms. This questionnaire also was conducted by KSNM based on the Rome III criteria for FD. ${ }^{13,15}$

To evaluate the degree of constipation, the Patient-Assessment of Constipation Symptoms (PAC-SYM) questionnaire ${ }^{16}$ was used. Three subscale scores for each domain (abdominal symptoms, rectal symptoms, and stool symptoms) and total scores were investigated according to the user guide. For the assessment of constipationassociated quality of life (QOL), the Patient-Assessment of Constipation Quality of Life (PAC-QOL) questionnaire ${ }^{17}$ was used. Four subscale scores for each domain (physical discomfort, psychosocial discomfort, worry and concerns, and satisfaction) and total scores were investigated according to the user guide. In addition, EQ-5D3L (EuroQol group, Rotterdam, Netherlands) questionnaire ${ }^{18,19}$ was used for the assessment of general health-related QOL. Data acquired from the descriptive system of EQ-5D-3L questionnaire were dichotomized into 'no problems' (ie, level 1) and 'problems' (ie, levels 2 and 3), and proportions of patients with 'problems' were assessed. EQ visual analogue scale (EQ VAS) was also evaluated to measure overall self-rated health status.

Korean versions of the above questionnaires were used after obtaining permission from their original copyright holders. For con- 
venience of presentation, the included patients were classified to 4 groups as follows according to the type of overlap: group I, patients with no overlap with other disorders; group II, patients presenting overlap with GERD; group III, patients presenting overlap with FD; and group IV, patients presenting overlap with both GERD and FD.

Table 1. Characteristics of Participants $(\mathrm{N}=759)$

\begin{tabular}{lc}
\hline Gender & \\
Male & $245(32.3 \%)$ \\
Female & $514(67.7 \%)$ \\
Age (mean $\pm \mathrm{SD}, \mathrm{yr})$ & $17.9(15-91)$ \\
Occupied area & \\
Capital area & $164(21.6 \%)$ \\
Chungchung/Gangwon & $93(12.3 \%)$ \\
Honam & $48(6.3 \%)$ \\
Kyeongbook & $216(28.5 \%)$ \\
Kyeongnam & $183(24.1 \%)$ \\
Jeju island & $55(7.2 \%)$ \\
Smoking history & \\
Nonsmoker & $568(74.8 \%)$ \\
Ex-smoker & $118(15.6 \%)$ \\
Smoker & $73(9.6 \%)$ \\
Drinking history & \\
Nondrinker & $330(43.5 \%)$ \\
Ex-drinker & $117(15.4 \%)$ \\
Drinker & $312(41.1 \%)$ \\
Underlying diseases & \\
Hypertension & $167(22.0 \%)$ \\
DM & $99(13.0 \%)$ \\
Diagnoses & \\
FC & $451(59.4 \%)$ \\
IBS-C & $308(40.6 \%)$ \\
\hline
\end{tabular}

$\mathrm{DM}$, diabetes mellitus; FC, functional constipation; IBS-C, irritable bowel syndrome with constipation.

\section{Statistical Methods}

The SPSS statistical package version 20.0 (IBM Corp, Armonk, NY, USA) was used for statistical analysis. All descriptive data are presented with the mean $\pm \mathrm{SD}$ (standard deviation) for continuous variables and with frequency or percentage for categorical variables. Risk factors affecting the overlap disorders were assessed by comparing group of patients with overlapping diseases with group of patients with non-overlapping diseases. Univariate analysis was performed with Student's $t$ test and chi-square test for continuous and categorical variables, respectively. Logistic regression analysis was performed to identify the independent risk factors related with the presence of overlap. One-way analysis of variance (ANOVA) test was performed to compare the mean values in 3 or more groups. After the ANOVA test, a Duncan test was performed as a post hoc test. $P$-values less than 0.05 were considered statistically significant.

\section{Results}

\section{Patient Demographics}

From 19 centers, 759 eligible subjects among 947 patients with constipation were included (Table 1). The proportion of males was $32.3 \%$ and the mean age was $52.3 \pm 17.9$ (range: $15-91$ ) years. The residential areas of the subjects were relatively evenly distributed throughout South Korea. Among the participants, 9.6\% and $41.1 \%$ were current smokers and drinkers, respectively. Furthermore, $22 \%$ and $13 \%$ of the subjects suffered from hypertension and DM, respectively. Of the 759 subjects, 451 (59.4\%) were diagnosed with IBS-C, and the other 308 (40.6\%) patients were diagnosed with FC.

Table 2. Prevalence of Overlaps

\begin{tabular}{|c|c|c|c|c|c|}
\hline & \multicolumn{4}{|c|}{ Group $^{a}$} & \multirow{2}{*}{ Total } \\
\hline & $\mathrm{I}^{\mathrm{b}}$ & II & III & $I V^{b}$ & \\
\hline \multicolumn{6}{|l|}{$\mathrm{FC}$} \\
\hline Frequency (n [\%]) & $314(69.6)$ & $72(16.0)$ & $43(9.5)$ & $22(4.9)$ & $451(100.0)$ \\
\hline \multicolumn{6}{|l|}{ IBS } \\
\hline Frequency (n [\%]) & $178(57.8)$ & $64(20.8)$ & $37(12.0)$ & $29(9.4)$ & $308(100.0)$ \\
\hline \multicolumn{6}{|l|}{ Total } \\
\hline Frequency (n [\%]) & $492(64.8)$ & $136(17.9)$ & $80(10.5)$ & $51(6.7)$ & $759(100.0)$ \\
\hline
\end{tabular}

${ }^{a}$ Grouping is as follows; group I, patients with no overlap with other disorders; group II, patients overlapped with gastroesophageal reflux disease (GERD); group III, patients overlapped with functional dyspepsia (FD); group IV, patients overlapped with both GERD and FD.

${ }^{\mathrm{b}}$ Significant differences between functional constipation $(\mathrm{FC})$ and irritable bowel syndrome (IBS) in the prevalence are found in group I $(P=0.001)$ and IV $(P=0.011)$. IBS-C, IBS with constipation. 


\section{Prevalence of Overlaps}

Overall, 492 (64.8\%) of the 759 subjects showed no overlap with GERD or FD (Group I). One hundred and thirty-six (17.9\%) presented co-incidental GERD (Group II), and 80 (10.5\%) presented co-incidental FD (Group III). Fifty-one (6.7\%) patients presented both co-incidental GERD and FD (Group IV) (Table 2).

Table 3. Univariate Analysis Between Overlapped and Non-overlapped Groups

\begin{tabular}{lccr}
\hline \multicolumn{1}{c}{ Variables } & $\begin{array}{c}\text { Overlap }(+) \\
(\mathrm{n}=267)\end{array}$ & $\begin{array}{c}\text { Overlap (-) } \\
(\mathrm{n}=492)\end{array}$ & $P$-value \\
\hline Male & $81(30.3 \%)$ & $164(33.3 \%)$ & 0.417 \\
Smoking & $23(8.6 \%)$ & $49(10.0 \%)$ & 0.546 \\
Drinking & $101(37.8 \%)$ & $211(42.9 \%)$ & 0.176 \\
Hypertension & $63(23.6 \%)$ & $104(21.1 \%)$ & 0.435 \\
DM & $37(13.9 \%)$ & $62(12.6 \%)$ & 0.624 \\
HNP & $9(3.4 \%)$ & $5(1.0 \%)$ & 0.021 \\
CVA history & $3(1.1 \%)$ & $5(1.0 \%)$ & 0.890 \\
Presence of pulmonary disease & $8(3.0 \%)$ & $4(0.8 \%)$ & 0.021 \\
Inadequate fiber intake & $65(24.3 \%)$ & $84(17.1 \%)$ & 0.016 \\
Ingestion of laxatives & $171(64.0 \%)$ & $243(49.4 \%)$ & $<0.001$ \\
Age (mean $\pm \mathrm{SD}$, years $)$ & $54.6 \pm 17.2$ & $51.1 \pm 18.2$ & 0.009 \\
BMI (mean $\left.\pm \mathrm{SD}, \mathrm{kg} / \mathrm{m}^{2}\right)$ & $22.6 \pm 3.1$ & $22.4 \pm 2.9$ & 0.428 \\
\hline
\end{tabular}

DM, diabetes mellitus; HNP, herniated nucleus pulposus; CVA, Cerebrovascular accident; BMI, body mass index.
When analyzed in isolation, $178(57.8 \%)$ of $308 \mathrm{C}-$ IBS patients and $314(69.6 \%)$ of 451 FC patients did not show overlapping GERD or FD. Therefore, the proportion of non-overlapped patients was higher in $\mathrm{FC}$ group $(P=0.001)$. Instead, $9.4 \%$ of IBS patients and $4.9 \%$ of $\mathrm{FC}$ patients presented overlapping with both GERD and FD, indicating higher prevalence of group IV in the IBS-C than the FC group $(P=0.011)$ (Table 2$)$.

\section{Risk Factors Associated with Overlap}

Of the various variables, the coincidence of $\operatorname{HNP}(P=0.021)$ or pulmonary diseases $(P=0.021)$, reduced fiber intake $(P=$ $0.016)$, laxative use $(P<0.001)$, and old age $(P=0.009)$ were positively associated with overlap (Table 3 ). Of these variables, (1) coincidental $\operatorname{HNP}(P=0.026)$ or pulmonary diseases $(P=0.034)$, (2) reduced fiber intake $(P=0.013)$, and (3) laxatives use $(P<$ $0.001)$ all independently affected the rate of overlap in multivariate analysis (Table 4).

\section{Degree of Symptoms Related to Constipation}

In the analyses of data from the PAC-SYM questionnaire, all 3 domains and the overall score showed significant differences in the degree of symptoms $(P<0.001)$ (Table 5$)$. Post hoc analyses with the Duncan test identified the patterns of these differences. In the domain of "abdominal symptoms," significant differences were noted among group I, group II and III, and group IV. There was

Table 4. Multivariate Analysis Between Overlapped and Non-overlapped Groups

\begin{tabular}{lllr}
\hline \multicolumn{1}{c}{ Variables } & Crude OR & Adjusted OR & $P$-value \\
HNP & $3.40(1.13-10.24)$ & $4.01(1.18-13.70)$ & 0.026 \\
Presence of pulmonary disease & $3.77(1.12-12.63)$ & $3.78(1.11-12.86)$ & 0.034 \\
Inadequate fiber intake & $1.57(1.09-2.26)$ & $1.61(1.10-2.34)$ & 0.013 \\
Ingestion of laxatives & $1.87(1.38-2.55)$ & $1.80(1.31-2.47)$ & $<0.001$ \\
Age & $1.01(1.00-1.02)$ & $1.00(0.99-1.01)$ & 0.538 \\
\hline
\end{tabular}

HNP, herniated nucleus pulposus.

Table 5. Degree of Symptoms Related to Constipation

\begin{tabular}{lcccccc}
\hline \multirow{2}{*}{ Domains } & \multicolumn{4}{c}{ Group $^{\mathrm{a}}$} & & \\
\cline { 2 - 5 } & I & II & III & IV & \\
\hline Abdominal symptoms & $1.01 \pm 0.69$ & $1.47 \pm 0.78$ & $1.42 \pm 0.79$ & $1.98 \pm 0.84$ & $<0.001$ \\
Rectal symptoms & $0.74 \pm 0.82$ & $0.94 \pm 0.90$ & $0.78 \pm 0.80$ & $1.24 \pm 0.93$ & $<0.001$ \\
Stool symptoms & $1.66 \pm 0.99$ & $2.02 \pm 0.96$ & $2.23 \pm 0.86$ & $2.54 \pm 0.93$ & $<0.001$ \\
Total score & $1.22 \pm 0.70$ & $1.57 \pm 0.74$ & $1.60 \pm 0.67$ & $2.01 \pm 0.78$ & $<0.001$ \\
\hline
\end{tabular}

${ }^{\mathrm{a}}$ Grouping is as follows; group I, patients with no overlap with other disorders; group II, patients overlapped with gastroesophageal reflux disease (GERD); group III, patients overlapped with functional dyspepsia (FD); group IV, patients overlapped with both GERD and FD.

${ }^{\mathrm{b}}$ The $P$-value in each domain was derived from one-way ANOVA test. 
Table 6. Constipation-associated Quality of Life According to Types of Overlap

\begin{tabular}{lccccc}
\hline \multicolumn{1}{c}{ Domains } & \multicolumn{4}{c}{ Group $^{\mathrm{a}}$} & \\
\cline { 2 - 5 } & I & II & III & IV & P-value \\
Physical discomfort & $1.74 \pm 0.86$ & $2.01 \pm 0.87$ & $2.34 \pm 0.94$ & $2.84 \pm 0.87$ & $<0.001$ \\
Psychosocial discomfort & $0.76 \pm 0.70$ & $1.21 \pm 0.83$ & $1.29 \pm 0.86$ & $1.85 \pm 1.04$ & $<0.001$ \\
Worries and concerns & $1.32 \pm 0.75$ & $1.72 \pm 0.85$ & $1.88 \pm 0.93$ & $2.23 \pm 1.02$ & $<0.001$ \\
Satisfaction & $2.58 \pm 0.76$ & $2.67 \pm 0.74$ & $2.87 \pm 0.77$ & $2.98 \pm 0.68$ & $<0.001$ \\
Total score & $1.44 \pm 0.59$ & $1.80 \pm 0.64$ & $1.96 \pm 0.72$ & $2.36 \pm 0.78$ & $<0.001$ \\
\hline
\end{tabular}

${ }^{a}$ Grouping is as follows; group I, patients with no overlap with other disorders; group II, patients overlapped with gastroesophageal reflux disease (GERD); group III, patients overlapped with functional dyspepsia (FD); group IV, patients overlapped with both GERD and FD.

${ }^{\mathrm{b}}$ The $P$-value in each domain was derived from one-way ANOVA test.

Table 7. Dichotomized Data From the Descriptive System of EQ-5D-3L Questionnaire According to Types of Overlap

\begin{tabular}{|c|c|c|c|c|c|c|}
\hline \multirow{2}{*}{ Domains } & \multirow{2}{*}{ Problem $(+)$} & \multicolumn{4}{|c|}{ Group $^{a}$} & \multirow{2}{*}{$P$-value } \\
\hline & & $\mathrm{I}(\mathrm{n}=474)$ & $\mathrm{II}(\mathrm{n}=134)$ & III $(\mathrm{n}=80)$ & $\operatorname{IV}(\mathrm{n}=51)$ & \\
\hline Exercise & Frequency (n [\%]) & $66(13.9)$ & $19(14.2)$ & $13(16.3)$ & $17(33.3)$ & 0.004 \\
\hline Self care & Frequency (n [\%]) & $19(4.0)$ & $12(9.0)$ & $6(7.5)$ & $11(21.6)$ & $<0.001$ \\
\hline Usual activity & Frequency (n [\%]) & $58(12.2)$ & $25(18.7)$ & $22(27.5)$ & $16(31.4)$ & $<0.001$ \\
\hline Pain/discomfort & Frequency (n [\%]) & $193(40.7)$ & $78(58.2)$ & $46(57.5)$ & $42(82.4)$ & $<0.001$ \\
\hline Anxiety/depression & Frequency (n [\%]) & $144(30.4)$ & $59(44.0)$ & $39(48.8)$ & $43(84.3)$ & $<0.001$ \\
\hline
\end{tabular}

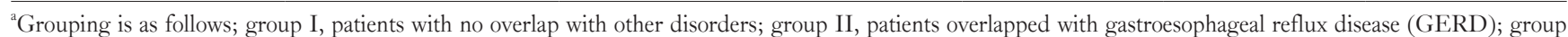
III, patients overlapped with functional dyspepsia (FD); group IV, patients overlapped with both GERD and FD.

The sample size of each group is slightly different from the original groups because of the missing values.

no difference between groups II and III. In the domain of "rectal symptoms," significant differences were noted only between group IV and other groups. We noted no difference among groups I, II, and III. In the domain of "stool symptoms" and "overall score," the patterns of differences were the same in "abdominal symptoms."

\section{Constipation-associated Quality of Life}

In the analyses of data from the PAC-QOL questionnaire, all 4 domains also showed significant differences in the degree of constipation-associated QOL $(P<0.001)$ (Table 6). Post hoc analyses with Duncan test also identified the patterns of these differences. In the domain of "physical discomfort," significant differences were noted among each of the independent groups. Domains of "psychosocial discomfort," "worry and concerns," "satisfaction," and "total score" showed the same patterns of differences. In this way, significant differences were noted among group I, groups II and III, and group IV, and there were no differences between groups II and III.

\section{General Health-related Quality of Life}

In the analyses of dichotomized data from the descriptive system of EQ-5D-3L questionnaire, proportions of patients with

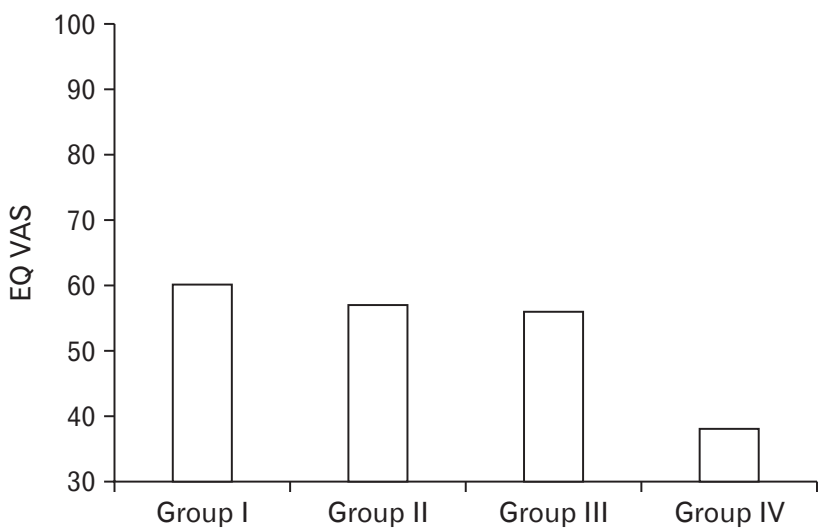

Figure. General health-related quality of life assessed with EQ visual analogue scale (EQ VAS) scoring. Poor quality of live is noted in group IV compared to group I, II, and III. Significant differences are noted only between group IV and other groups, while there are no differences among group I, II, and III. Grouping is as follows: group I, patients with no overlap with other disorders; group II, patients overlapped with gastroesophageal reflux disease (GERD); group III, patients overlapped with functional dyspepsia (FD); group IV, patients overlapped with both GERD and FD. 
"problem" were significantly higher in all 5 domains (exercise, selfcare, usual activity, pain/discomfort, and anxiety/depression) in groups of patients presenting overlapping conditions, especially in group IV (Table 7). The mean EQ-day scores in EQ VAS system from group I to group IV were 60.1, 57.1, 56.0, and 38.1, respectively. Therefore, significant differences were noted $(P<0.001)$ (Figure). Post hoc analyses with the Duncan test identified that significant differences were noted only between group IV and other groups, while there were no differences among group I, II, and III.

\section{Discussion}

In this study, the patterns of overlap of two common FGIDs (ie, symptomatic GERD and FD) in patients with constipation were investigated. We also investigated how these overlaps affect the quality of life in constipated patients. Our results show that more than $30 \%$ of constipating patients present overlap of one or both investigated diseases, and that QOL of these patients is poorer than that of patients who do not present overlapping diseases.

Constipation is a type of frequent GI symptom in which the prevalence has been reported to be up to $20 \%$ depending on regions. ${ }^{20,21}$ "Constipation" as a symptom has comprehensive meaning depending on the person. It includes various states, such as a sense of insufficient defecation, propulsive force during defecation, sense of barriers of defecation, longer stay in the bathroom, hard stool, and decreased frequency of defecation. ${ }^{22}$ In our study, we considered patients who visited outpatient clinics complaining difficult, infrequent, or incomplete defecation as primary subjects of the study.

The Korean version of the self-administered symptom questionnaire ${ }^{13}$ based on the Rome III criteria ${ }^{12}$ (Rome III-K) was used in our study to define FC, IBS-C, and FD. Rome III-K is composed of 35 questions that assess the presence or frequency of various GI symptoms. It was validated linguistically by KSNM in 2013, and is useful for the diagnosis of functional heartburn, FD, IBS, FC, functional diarrhea, and functional bloating.

We enrolled the patients in this study if he or she fulfilled the Rome III criteria for FC or IBS-C and had no organic disorders. Although patients who had obvious organic disorders such as acute abdomen, intestinal obstruction, intestinal pseudo-obstruction, and malignancy were excluded, complete exclusion of secondary constipation was impossible due to limitations of the observational questionnaire study. Although the Rome III and even Rome IV criteria for functional bowel disorders suggest that IBS-C and FC are mutually exclusive, there are several studies reporting that these conditions are not completely different. ${ }^{23-25}$ In a recent systematic review, it was found that most constipated patients meet the criteria for both IBS-C and FC, if the Rome III requirement for FC "Insufficient criteria for IBS" is suspended. ${ }^{26}$ Additionally, contrary to perceived expectations, IBS-C patients had more constipationassociated symptoms rather than patients with FC. Since the clearcut discrimination between IBS and FC is often difficult in real clinical situations, we enrolled and analyzed both FC and IBS-C patients without dividing in our study. Discrimination between IBS and $\mathrm{FC}$ was performed only to determine the prevalence of overlapping conditions.

Although various FGIDs are structured to be diagnosed mutually exclusively in the Rome III criteria, the possibility of overlap among the FGIDs can be easily predicted considering the common pathophysiology of these disorders. There have been several original or review reports on the overlap of these FGIDs, particularly among common FGIDs such as FD, symptomatic GERD, and IBS. ${ }^{3-8,10,11,27-29}$ According to a review article conducted by Fujiwara and Arakawa, ${ }^{5}$ up to $15.8 \%$ of the general population and $62.3 \%$ of outpatients have been reported to have overlap between both subtypes of FD (ie, epigastric pain syndrome and postprandial distress syndrome). Furthermore, the overlap of reflux symptoms in patients with uninvestigated dyspepsia has been reported to vary from $13.3 \%$ to $63.0 \%$ of prevalence. ${ }^{5}$ In another systematic review, the prevalence of IBS in patients with dyspepsia was reported to be $37.0 \%$ compared to $7.0 \%$ in those without dyspepsia. ${ }^{10}$ Therefore, the odds ratio for IBS in patients with dyspepsia was about 8.00 (95\% CI, 5.74-11.16). As well as FGIDs, other functional disorders including chronic fatigue syndrome, fibromyalgia, and hypersensitive bladder are known to coexist with FD and IBS in the same patient. ${ }^{30}$ Although there is a limitation that the sample size is relatively small, the fact that overlap is common among FGIDs has also been reported in an Asian study. ${ }^{27}$

Our study shows that patients with constipation frequently overlap with GERD, FD, or both. However, the patterns of overlaps are somewhat different from previous reports. Unlike other functional disorders, studies about overlap in patients with constipation is rare. In a systematic review which analyzed 35 studies about comorbid conditions in IBS-C and chronic idiopathic constipation, $\mathrm{FD}$ and depression were reported as the most common comorbid diseases in patients with IBS-C, whereas FD, DM, and depression were the most commonly overlapping diseases in patients with chronic idiopathic constipation. ${ }^{31}$ In another web-based, crosssectional study conducted in the United States, the impact of overlapping FD, GERD, IBS-C, and FC on symptom burden and consulting behavior was assessed. ${ }^{32}$ Of the 2641 responders, 
328 and 552 persons met the Rome III criteria for IBS-C or FC, respectively. In their study, only $17.4 \%$ of IBS and $37.6 \%$ of $\mathrm{FC}$ existed alone without overlapping GERD or FD (this group corresponds to group I in our study). These fractions were lower than our study which showed $57.8 \%$ and $69.6 \%$, respectively. The portions of IBS and FC patients overlapping with GERD (corresponds to group II in our study) in their study were $7.3 \%$ and $10.1 \%$, respectively, which were also lower than our study of $20.8 \%$ and $16.0 \%$, respectively. However, the portions of patients presenting overlap with FD (corresponds to group III in our study) in IBS and FC were $40.8 \%$ and $33.5 \%$, respectively. These values were significantly higher than our study, which measured rates of $12.0 \%$ and $9.5 \%$, respectively. Similarly, the portions of patients presenting overlap with both GERD and FD (corresponds to group IV in our study) were $34.5 \%$ in IBS and $18.8 \%$ in FC. These values were also higher than our study, which measured rates of only $9.4 \%$ and $4.9 \%$, respectively. The overall overlap frequency and overlap frequency with $\mathrm{FD}$ were low; however, the overlap frequency with GERD was high in our study. There was a similar tendency in that the overall overlap frequency was higher in the IBS patients than in the FC patients. It is uncertain whether these differences are due to regional differences (Eastern vs Western) or due to differences in the nature of participants (patients who visited hospital vs general population who responded web-based questionnaire).

We found that the rate of overlap is associated with coincidental HNP or pulmonary diseases, reduced fiber intake, and laxatives use. Several independent risk factors have been suggested in relation to overlapping FGIDs. Self-reported insomnia, frequent abdominal pain, higher somatization score, and higher body mass index have been presented as risk factors for overlap of IBS and symptomatic GERD in a population study. ${ }^{33}$ Insomnia and somatization also have been suggested as risk factors for the overlap of FD and GERD in another study. ${ }^{7}$ Similarly, the presence of depression has been reported to be a significant associated factor in the overlap of FD and IBS. ${ }^{34}$ Therefore, a wide variety of variables may act as risk factors for the overlap of several FGIDs, depending on the variables to be considered. In this study, the co-incidence of HNP or pulmonary diseases, reduced fiber intake, and laxatives use independently affected the overlap of constipation with disorders discussed in this study. However, it is not appropriate to judge these variables as independent risk factors based solely on these data for several reasons. First, several variables that were considered as risk factors for overlap in other studies were not included in the questionnaire used in this study. Second, because the presence of HNP or pulmonary diseases was investigated with open questions and not a closed question, the reliability is low. Furthermore, fiber intake also was not quantitatively measured. Next, laxative use was more likely to be a result of severe symptoms in patients with overlapping diseases rather than to be a risk factor itself. Therefore, to obtain accurate independent risk factors associated with overlap, well-designed questionnaires that consider various variables will be necessary.

This study shows that the symptoms of patients with overlaps are more severe than those of patients without overlap. It is known from several reports that the symptom burdens of FGIDs are heavier when one disorder overlaps with one or more disorders. ${ }^{5,6,9,10,29}$ This tendency also applies to patients with constipation, as in previous study. ${ }^{32}$ In our study, we assessed the degree of constipation using a well-known PAC-SYM questionnaire. This questionnaire contains 12 items assigned to 3 subscales: stool symptoms ( 5 items), rectal symptoms ( 3 items), and abdominal symptoms (4 items). Each item is scored using a 5 -point Likert scale (from 0 to 4), with lower scores meaning less symptom severity. Scores of $0,1,2,3$, and 4 mean absent, mild, moderate, severe, and very severe, respectively. As predicted, this study showed that symptoms of two (abdominal symptoms and stool symptoms) domains as well as overall symptoms were more severe in patients with overlapping diseases with GERD or FD. Furthermore, the symptom severity was the highest in patients with all three combined disorders. Although there was no difference in the symptom severity of rectal symptoms between the group without overlap and the group with overlap with one other disease, the symptom severity of this domain also was the highest in patients with all three combined disorders.

As expected, poorer QOL was observed in patients with overlaps compared to patients without overlap in this study. It is not difficult to predict that the quality of life will be lower with more severe symptoms in the overlapping groups, and there are several reports on this. ${ }^{3,5,6,31}$ In this study, we assessed both constipation-associated QOL and general health-related QOL with PAC-QOL and EQ5D-3L questionnaires, respectively. PAC-QOL questionnaire contains 28 items assigned to 4 domains: worries and concerns (11 items), physical discomfort (4 items), psychosocial discomfort (8 items), and satisfaction ( 5 items). Each item was also scored using a 5 -point Likert scale (from 0 to 4 ) with a lower score meaning a higher QOL although several items required a scoring revision. This study showed that constipation-associated QOL of all domains were lower in patients with overlapping diseases that included either GERD or FD. These scores were the lowest in patients combined with all 3 disorders. The EQ-5D-3L questionnaire consisted of the EQ-5D descriptive system and the EQ VAS. The EQ-5D- 
3L descriptive system contains 5 dimensions: mobility, self-care, usual activities, pain/discomfort, and anxiety/depression. Although each dimension is scored using 3 levels, we dichotomized them into 'no problems' and 'problems' for convenience according to the user guide. As expected, the proportion of patients with disability was significantly higher in the group of patients with overlapping diseases in all 5 dimensions. Similarly, EQ VAS revealed that lowest QOL in in patients combined with all three disorders.

There were several limitations to this study. First, despite various medications and diseases that cause secondary constipation, we could not investigate these factors accurately and precisely. This limitation is more prominent, especially since most of the questions about these factors were designed as open questions. Second, due to the limitations of large-scale questionnaire studies, the results of additional tests on constipation subtypes were not reflected. Finally, regarding the degree of symptoms and the quality of life, FC and IBS-C were not separately analyzed. A larger sample size is needed for this analysis. Therefore, to overcome the above limitations, a cohort study including data collection by common clinical pathways for a sufficient period is necessary. Additionally, for the detection of risk factors associated with overlaps, it is necessary to investigate the presence of various diseases related to constipation through closed questions and objective clinical data.

Despite these limitations, this study is worthy as it is a rare study that reflects the frequency, symptom severity, and even quality of life about overlapping FGIDs in patients with constipation in that data were collected prospectively by various institutions in various regions.

\section{Supplementary Material}

Note: To access the supplementary table mentioned in this article, visit the online version of Journal of Neurogastroenterology and Motility at http://www.jnmjournal.org/, and at https://doi. org/10.5056/jnm17033.

Financial support: This work was funded by Jeil Pharmaceutical grants from The Korean Society of Neurogastroenterology and Motility, Korea (2013), and also supported by Wonkwang University in 2016 (Suck Chei Choi).

\section{Conflicts of interest: None.}

Author contributions: Kyung Sik Park: study design, data acquisition, data analysis, data interpretation, and writing the manuscript; Suck Chei Choi: study design, data acquisition, data analysis, data interpretation, and drafting the manuscript; and Sam Ryong Jee, Bong Eun Lee, Kyoung Sup Hong, Jeong Eun Shin, Soo-Young $\mathrm{Na}$, Joong Goo Kwon, Yong Sung Kim, Hyun Seok Lee, Tae Hee Lee, Kyeong Ok Kim, Jongkyoung Choi, Hee Seok Moon, Yeon Soo Kim, Moo In Park, Soo Jung Park, Seon-Young Park, and Sung Noh Hong: data acquisition and critical revision of the manuscript.

\section{References}

1. Drossman DA. Functional gastrointestinal disorders: history, pathophysiology, clinical features and Rome IV. Gastroenterology Published Online First: 19 Reb 2016. doi: 10.1053/j.gastro.2016.02.032.

2. Drossman DA. The functional gastrointestinal disorders and the Rome III process. Gastroenterology 2006;130:1377-1390.

3. Kim SE, Chang L. Overlap between functional GI disorders and other functional syndromes: what are the underlying mechanisms? Neurogastroenterol Motil 2012;24:895-913.

4. Choung RS. [Natural history and overlap of functional gastrointestinal disorders.] Korean J Gastroenterol 2012;60:345-348. [Korean]

5. Fujiwara Y, Arakawa T. Overlap in patients with dyspepsia/functional dyspepsia. J Neurogastroenterol Motil 2014;20:447-457.

6. de Bortoli N, Martinucci I, Bellini M, et al. Overlap of functional heartburn and gastroesophageal reflux disease with irritable bowel syndrome. World J Gastroenterol 2013;19:5787-5797.

7. Choung RS, Locke GR 3rd, Schleck CD, Zinsmeister AR, Talley NJ. Overlap of dyspepsia and gastroesophageal reflux in the general population: one disease or distinct entities? Neurogastroenterol Motil 2012;24:229-234, e106.

8. Suzuki H, Hibi T. Overlap syndrome of functional dyspepsia and irritable bowel syndrome - are both diseases mutually exclusive? J Neurogastroenterol Motil 2011;17:360-365.

9. Kaji M, Fujiwara Y, Shiba M, et al. Prevalence of overlaps between GERD, FD and IBS and impact on health-related quality of life. J Gastroenterol Hepatol 2010;25:1151-1156.

10. Ford AC. Overlap among the functional gastrointestinal disorders. Am J Gastroenterol 2010;105:2512.

11. Lee SY, Lee KJ, Kim SJ, Cho SW. Prevalence and risk factors for overlaps between gastroesophageal reflux disease, dyspepsia, and irritable bowel syndrome: a population-based study. Digestion 2009;79:196-201.

12. Longstreth GF, Thompson WG, Chey WD, Houghton LA, Mearin F, Spiller RC. Functional bowel disorders. Gastroenterology 2006;130:1480-1491.

13. Song KH, Jung HK, Min BH, et al. Development and validation of the Korean Rome III questionnaire for diagnosis of functional gastrointestinal disorders. J Neurogastroenterol Motil 2013;19:509-515.

14. Jones R, Junghard O, Dent J, et al. Development of the GerdQ, a tool for the diagnosis and management of gastro-oesophageal reflux disease in primary care. Aliment Pharmacol Ther 2009;30:1030-1038.

15. Tack J, Talley NJ, Camilleri M, et al. Functional gastroduodenal disor- 
ders. Gastroenterology 2006;130:1466-1479.

16. Frank L, Kleinman L, Farup C, Taylor L, Miner P Jr. Psychometric validation of a constipation symptom assessment questionnaire. Scand J Gastroenterol 1999;34:870-877.

17. Marquis P, De La Loge C, Dubois D, McDermott A, Chassany O. Development and validation of the Patient Assessment of Constipation Quality of Life questionnaire. Scand J Gastroenterol 2005;40:540-551.

18. Brooks R. EuroQol: the current state of play. Health Policy 1996;37:5372.

19. EuroQol Group. EuroQol--a new facility for the measurement of healthrelated quality of life. Health Policy 1990;16:199-208.

20. Jun DW, Park HY, Lee OY, et al. A population-based study on bowel habits in a Korean community: prevalence of functional constipation and self-reported constipation. Dig Dis Sci 2006;51:1471-1477.

21. Talley NJ, Weaver AL, Zinsmeister AR, Melton LJ 3rd. Functional constipation and outlet delay: a population-based study. Gastroenterology 1993;105:781-790.

22. Drossman DA, Li Z, Andruzzi E, et al. U.S. householder survey of functional gastrointestinal disorders. Prevalence, sociodemography, and health impact. Dig Dis Sci 1993;38:1569-1580.

23. Rey E, Balboa A, Mearin F. Chronic constipation, irritable bowel syndrome with constipation and constipation with pain/discomfort: similarities and differences. Am J Gastroenterol 2014;109:876-884.

24. Shekhar C, Monaghan PJ, Morris J, et al. Rome III functional constipation and irritable bowel syndrome with constipation are similar disorders within a spectrum of sensitization, regulated by serotonin. Gastroenterology 2013;145:749-757.

25. Wong RK, Palsson OS, Turner MJ, et al. Inability of the Rome III criteria to distinguish functional constipation from constipation-subtype irritable bowel syndrome. Am J Gastroenterol 2010;105:2228-2234.

26. Siah KT, Wong RK, Whitehead WE. Chronic constipation and constipation-predominant IBS: separate and distinct disorders or a spectrum of disease? Gastroenterol Hepatol (N Y) 2016;12:171-178.

27. Xiong LS, Shi Q, Gong XR, Cui Y, Chen MH. The spectra, symptom profiles and overlap of Rome III functional gastrointestinal disorders in a tertiary center in South China. J Dig Dis 2014;15:538-544.

28. Shin CM. Overlap between postprandial distress and epigastric pain syndromes in functional dyspepsia: its implications for research and clinical practice (Am J Gastroenterol 2013;108:767-774). J Neurogastroenterol Motil 2013;19:409-411.

29. Park H. Functional gastrointestinal disorders and overlap syndrome in Korea. J Gastroenterol Hepatol 2011;26(suppl 3):12-14.

30. Henningsen P, Zipfel S, Herzog W. Management of functional somatic syndromes. Lancet 2007;369:946-955.

31. Nellesen D, Chawla A, Oh DL, Weissman T, Lavins BJ, Murray CW. Comorbidities in patients with irritable bowel syndrome with constipation or chronic idiopathic constipation: a review of the literature from the past decade. Postgrad Med 2013;125:40-50.

32. Vakil N, Stelwagon M, Shea EP, Miller S. Symptom burden and consulting behavior in patients with overlapping functional disorders in the US population. United European Gastroenterol J 2016;4:413-422.

33. Jung HK, Halder S, McNally M, et al. Overlap of gastro-oesophageal reflux disease and irritable bowel syndrome: prevalence and risk factors in the general population. Aliment Pharmacol Ther 2007;26:453-461.

34. Lee HJ, Lee SY, Kim JH, et al. Depressive mood and quality of life in functional gastrointestinal disorders: differences between functional dyspepsia, irritable bowel syndrome and overlap syndrome. Gen Hosp Psychiatry 2010;32:499-502. 